\title{
Vitality Detection from Fingerprint Images: A Critical Survey
}

\author{
Pietro Coli, Gian Luca Marcialis, and Fabio Roli \\ University of Cagliari - Department of Electrical and Electronic Engineering \\ Piazza d'Armi - 09123 Cagliari (Italy) \\ \{pietro.coli, marcialis, roli\}@diee.unica.it
}

\begin{abstract}
Although fingerprint verification systems reached a high degree of accuracy, it has been recently shown that they can be circumvented by "fake fingers", namely, fingerprint images coming from stamps reproducing an user fingerprint, which is processed as an "alive" one. Several methods have been proposed for facing with this problem, but the issue is far from a final solution. Since the problem is relevant both for the academic and the industrial communities, in this paper, we present a critical review of current approaches to fingerprint vitality detection in order to analyze the state-of-the art and the related open issues.
\end{abstract}

\section{Introduction}

In the last years, fingerprint verification systems for personal identity recognition reached a high degree of accuracy [1]. Fingerprints can be reasonably considered the biometric for which academic and industrial research achieved the highest level of maturity. In fact, many capture devices have been implemented with powerful software development kits for acquiring, processing and matching fingerprint images. The reason of this success is mainly due to the most claimed characteristic of fingerprints: their uniqueness [2]. In other words, it is claimed that fingerprints are unique from person to person, and the probability to find two similar fingerprints characterized, for example., by minutiae is very low [2].

However, recent works pointed out that the current fingerprint capture devices can be deceived by submitting a "fake fingerprint" made up of gelatine or liquid silicon [3]. This fake finger can be obtained by person coercion (the so-called "consensual" method) or by latent fingerprints [3]. The first method is the most simple, as it requires that the person put his finger on a plasticine-like material. The next step is to drip over this mould some liquid silicon. After its solidification, a fake stamp reproducing the fingerprint of the client can be used for deceiving the acquisition sensor, which processes that as an "alive" fingerprint. An example of "live" and "fake" fingerprint images fabricated by the consensual method and acquired with an optical sensor is given in Figure 2. Obviously, reproducing a fingerprint is not so easy as it may appear, because high quality stamps are necessary for a successful logon on a system protected with a fingerprint authentication module. On the other hand, the issue exists, as it was pointed out in [3]. 
Since fingerprints can be reproduced, this has a dramatic impact on the main property which made them so popular and appealing for security applications: their uniqueness. The state-of-the-art literature addresses this crucial problem as follows: is the finger on the acquisition sensor alive or "fake" ? In order to handle this problem, several methods to detect the fingerprint vitality (or "liveness") have been proposed, and this research field is still very active. A first subdivision of the state-of-the-art approaches can be made by observing that vitality is detected by extracting vitality measures from the finger, as the heartbeat or the blood pressure (hardware-based approaches), or from the fingerprint image(s) directly (software-based approaches).

As, to our knowledge, no previous paper reviewed critically the state-of-the-art of fingerprint vitality detection methods, in this paper, we propose a taxonomy of current methods (Section 2) and discuss and compare some key features of previous works, such as the material used for fingerprint reproduction and the data sets used (Section 3). In section 4, we analyze and compare the detection performances reported in the literature. Some conclusions are drawn in Section 5.

\section{Fingerprint Vitality Detection: A Taxonomy of Existing Methods}

A possible taxonomy of fingerprint vitality detection methods is proposed in Figure 1. Roughly, existing approaches can be subdivided in "hardware-based" and "softwarebased". The first ones try to detect the vitality of the fingertip put on the sensor by additional hardware able to measure, for example, blood pressure [4], heartbeat [5], fingertip odor [6], or skin impedance [7]. These approaches are obviously expensive as they require additional hardware and can be strongly invasive: for example, measuring person's blood pressure is invasive as it can be used for other reasons that for simply detecting the vitality of his fingertip [8]. Moreover, in certain cases a clever imitator can circumvent these vitality detection methods.

Therefore, making the image processing module more "intelligent", that is, making it able to detect if a fake finger has been submitted is an interesting alternative to the hardware-based approaches. Several approaches aimed to extract vitality features from the fingerprint images directly have been recently proposed [9-15]. The general rationale behind these approaches is that some peculiarities of "live fingerprints" cannot be hold in artificial reproductions, and they can be detected by a more or less complex analysis of fingerprint images. The related vitality detection approaches can be named "software-based".

In this survey, we focus on the software-based approaches. Therefore, in the taxonomy of Figure 1, the leave associated to hardware-based approaches is not expanded further.

According to the taxonomy of Figure 1, the initial subdivision of the softwarebased approaches is based on the kind of features used. If the features extracted derive from the analysis of multiple frames of the same image, captured while the subject puts his fingertip on the acquisition surface at certain time periods (e.g., at $0 \mathrm{sec}$ and at $5 \mathrm{sec}$ ), the related methods are named "dynamic" (as they use dynamic features). 
On the other hand, if features are extracted from a single fingerprint impression or the comparison of different impressions, the methods are named "static"(as they use static features).

Referring to the leaves of the taxonomy in Figure 1, they describe software-based approaches as functions of the physical principle they exploit: the perspiration, the elastic distortion phenomena, and the intrinsic structure of fingerprints (morphological approaches).

According to the proposed taxonomy, in the following sections, we review the vitality detection methods proposed in the scientific literature.

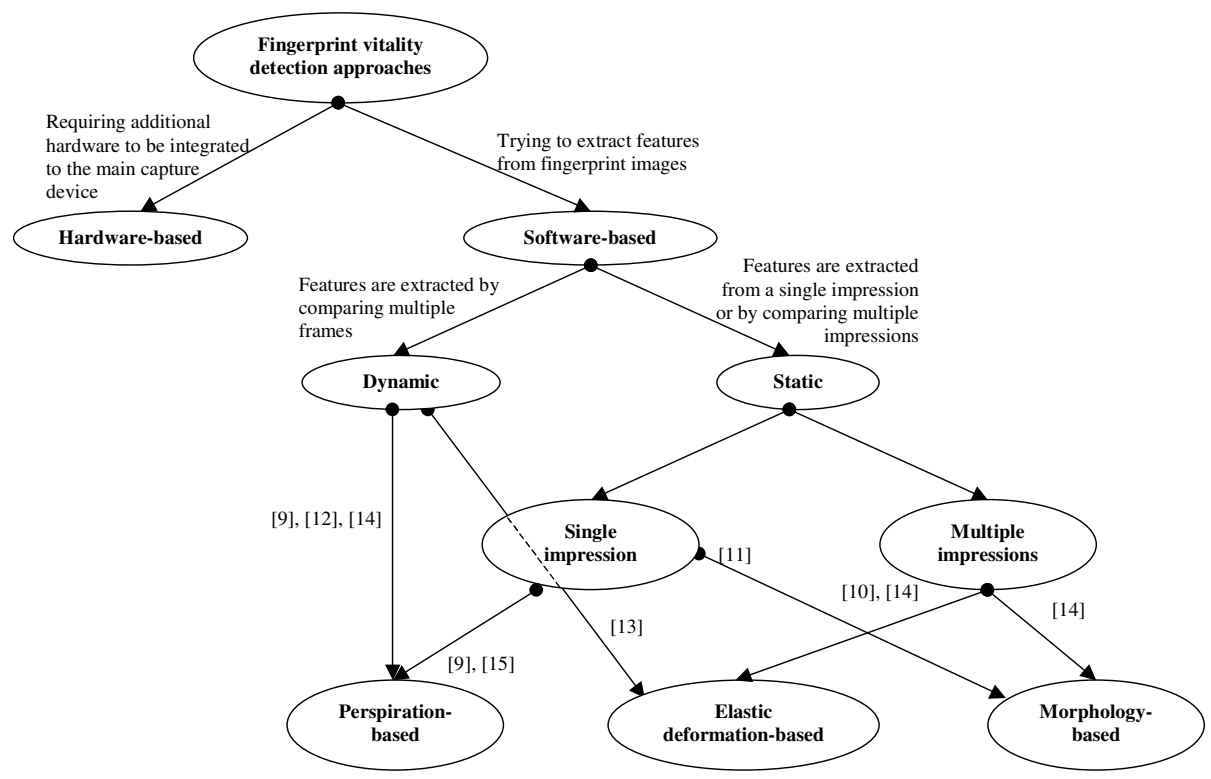

Fig. 1. The proposed taxonomy of fingerprint vitality detection methods. Labels of the edges in the form [*] are the number of the related Reference.

\subsection{Static Methods}

\subsubsection{Static Methods Using a Single Impression}

Following the path of the tree in Figure 1 from the static methods-junction, we first consider methods which exploit single impression. These can be classified into two further classes: perspiration and morphology based. About the former we have selected two main works as [15] and [9]. Both study the perspiration phenomenon with two transforms, [15] with wavelet space, [9] with Fourier space. Tan and Schuckers [15] showed how it is possible to clearly distinguish a live from a fake finger by wavelet transform. The rationale of this method is the analysis of the particular shape of the finger surface. In live fingers, in order to guarantee the physiological thermo-regulation, there are many little chinks named "pores" scattered along the center of the ridges. Because of this characteristic the acquired image of a finger shows a non-regular shape of the ridges. Generally the path of the ridges is 
irregular and, if the resolution of the device is high enough, it is possible to observe these pseudo-periodic conformations at the center of the ridges. With the fabrication step of an artificial finger it is possible to lose these micro-details and consequently the correspondent acquired image is more regular in the ridge shape. The authors propose to analyze this feature with a wavelet decomposition. In particular, the image is enhanced and converted into a mono-dimensional signal as the gray level profile extracted in correspondence of the center of the ridges. A wavelet decomposition of this signal is applied with a five-levels multiresolution scheme: The standard deviation, the mean value for each wavelet coefficient and from the original and the last approximation signals are computed. The obtained 14 parameters are considered as a feature-vector for the next classification stage.

The concept of detecting liveness from the skin perspiration analysis of the pores has been already proposed in [9]. In particular, the authors uses one static feature, named $S M$, based on the Fast Fourier Transform of the fingerprint skeleton converted into a mono-dimensional signal. The rationale is that for a live finger it is possible to notice clearly the regular periodicity due to the pores on the ridges. On the contrary this regularity is not evident for spoof fingerprint signals.

Carrying on with single impression-static methods, another work is noticeable to mention. Unlike the previous works, this applies a liveness detection studying the morphology of the fingerprint images. So, referring to Figure 1, the branch ending to morphologic based method is related to the work by Moon et al. [11].

The study is based on a different method with a contrasting argument. Looking at the finger surface with an high resolution Digital Single Lens Reflex camera, they observe that the surface of a fake finger is much coarser then that of a live finger. The main characteristic of this work is that an high resolution sensor is necessary for successfully capturing this difference (1000 dpi, whilst current sensors exhibit 500 dpi on average). Moreover, this approach does not work with the entire image, too large because of its resolution, but with subsamples of a fixed size. For extracting this feature, the residual noise returned from a denoising-process applied to the original sub-images is considered. The standard deviation of this noise is then computed to highlight the difference between live and fake coarseness.

\subsubsection{Static Methods Using Multiple Impressions}

Whilst the previous studies search a liveness indication from intrinsic properties of a single impression, there are other static features based on multiple impressions: in this case the liveness is derived from a comparison between a reference template image and the input image. This methods are represented in Figure 1 with two branches starting from the "Multi-impressions" node: one indicates method based on elasticdeformation features, the other indicates ones based on morphologic features. Ref [10] falls within the first category. Given a genuine query-template pair of fingerprints, the entity of elastic distortion between the two sets of extracted minutiae is measured by a thin-plate spline model. The idea is that live and spoof fingerprints show different elasticity response repeating the acquisitions.

The experimental investigation of Coli et al. [14] considers both the elasticdeformation based method and the morphology-based one. The elastic deformation is evaluated by computing the averaged sum of all the distances among the matched 
minutiae of input and template fingerprints. The different elastic response of a live finger or an artificial stamp is linked with the spread of this mean value. The other static multi-impression measure is based on morphologic investigation. The feature that makes use of the ridge width is based on the idea that during the creation of fingerprint replica, there is an unavoidable modification of the thickness of the ridges: first when the user put his finger on the cast material, next when the stamp is created with liquid silicone.

\subsection{Dynamic Methods}

Dynamic methods for vitality detection relies on the analysis of different image frames acquired during an interval while the user put his finger on the scanner.

As it is schematized in Figure 1 there are two types of dynamic methods: one based on the perspiration phenomenon, the other on the elastic response of the skin.

The same properties of the skin used for a static measure in $[9,11]$ is exploited with dynamic analysis based on the first approach [9]: the pores scattered on the fingertip surface are the source of the perspiration process. When the finger is in contact with the surface of the scanner, the skin gets wetter because of an increasing of sweat amount. This physiological phenomenon can be recorded by acquiring sequential frames during a fixed interval of few seconds. The variation of the wetness of the fingertip skin reflects on a variation of the gray-level profile of the acquired images. In order to evaluate this feature, the fingerprint skeleton of the image at 0 and 5 seconds is converted into a couple of mono-dimensional signals $(C 1, C 2)$. Several statistical measures are proposed on the basis of the obtained signals. In particular [9], DM1 ( Total swing ratio ), DM2 (Min/Max growth ratio), DM3 (Last-First fingerprint signal difference mean), DM4 (Percentage change of standard deviation).

By considering that Ref. [12] draws up a more complete vitality analysis on different technology of fingerprint scanner, it has been necessary to introduce some modifications to the original method. The dynamic of the device can produce a saturated signal for excessive amount of wetness, in such situation the feature DM2 lost its original efficacy. In order to avoid this drawback two new features named DM5 (Dry saturation percentage change) and DM6 (Wet saturation percentage change) are elaborated. With a selection of these measures, Coli et al. [14] applies a liveness detection on their extended database.

As the previous works can be considered an extension of the static perspiration measures, the work of Antonelli et Al. [13] adopts a dynamic procedure in order to perform a liveness detection based on elastic deformation. The user holding his finger on the scanner surface is invited to apply a rotation of the fingertip. The movement of the fingertip on the surface induces an elastic tension to the whole surface and consequently an elastic deformation depending on the level of elasticity of the skin, for live finger, or of the artificial material for the spoof stamp. A dynamic acquisition extracts a sequence of images at high frame rate ( $>20 \mathrm{fps})$. The authors named "distortion-code" the feature-vector encoding the elastic distortion from the current frame to the next one, obtained computation of the optical flows that estimates the variation in the time of the rotating images. 


\section{Previous Works: Fabrication Process of Fake Stamps and Data Sets Used}

We believe that a critical review of previous works on fingerprint vitality detection should analyze: (i) the different materials employed for fake stamps and the methods used for creating them; (ii) the characteristics of the data sets used for the vitality detection experiments.

Table 1 deals with Item (i) for the methods we reviewed in Section 2. Item (i) is important because the response of a certain fingerprint scanner varies with the material adopted (e.g., gelatine or silicon). Secondly, the intrinsic quality of the stamp depends on the material for the cast and the method followed for its creation. With regard to the mould materials, all those employed are able to deceive an optical sensor, as pointed out in [3]. On the other hand, using the silicone material is not effective for capacitive sensors, probably due to the different electrical properties of this material with respect to the skin.

Table 1. Software-based methods for fingerprint vitality detection and materials and methods used for stamp fabrication

\begin{tabular}{|l|l|l|l|l|}
\hline Reference & Scanner & Method & Cast material & Mould material \\
\hline$[9]$ & Capacitive & Consensual & Rubber & Play-Doh \\
\hline$[10]$ & Not specified & Consensual & Gum & Gelatine \\
\hline$[11]$ & Digital cam. & Consensual & Not specified & $\begin{array}{l}\text { Gelatine } \\
\text { Plastic clav. }\end{array}$ \\
\hline$[13]$ & Optical & Consensual & Not specified & $\begin{array}{l}\text { Silicone, } \\
\text { Gelatine, } \\
\text { Latex }\end{array}$ \\
\hline$[12]$ & $\begin{array}{l}\text { Optical } \\
\text { Electro-optical } \\
\text { Capacitive }\end{array}$ & Consensual & $\begin{array}{l}\text { Dental } \\
\text { impression }\end{array}$ & Play-Doh \\
\hline$[14]$ & Optical & Consensual & Plasticine & Silicone \\
\hline$[15]$ & $\begin{array}{l}\text { Optical } \\
\text { Electro-optical } \\
\text { Capacitive }\end{array}$ & Consensual & Not specified & $\begin{array}{l}\text { Play-Doh } \\
\text { Gelatine }\end{array}$ \\
\hline
\end{tabular}

In table 1 it is worth noting that all the approaches at the state-of-the-art used the consensual method for creating the stamp. This method consists in three steps: (1) the user put his finger on a mould material: the pattern of the fingerprint image negative is reproduced in a mould; (2) the cast material (e.g. liquid silicon with a catalyst) is dripped over the mould: the liquid covers the negative fingerprint; (3) after some hours the solidification of the rubber is completed and the cast can be removed from the mould; (4) the rest of the mould is cleaned off from the surface of the cast. This method exploits the subject cooperation whilst other approaches, e.g., the ones that produce a fake stamp from latent fingerprints, are more complex and requires more expert knowledge [3]. Moreover, the quality of stamps is intrinsically lower than that obtained with the consensual method. The consensual method is used for obtaining high quality stamps, in order to create a severe performance test for vitality detection. 
It is, in fact, easy to see that fraudulent attacks using high quality stamps are much more difficult to detect, while attacks with low quality stamps can be, in many cases, detected also without using a vitality detection module (the fake impression is rejected as an impostor impression).

However, it should be noted that several variables are involved in the fraudulent access process, in particular: (1) the initial pressure of the subject on the cast; (2) the mould material dripped over the cast; (3) the contact of the stamp on the acquisition surface. These variables concur to alter the shape of the reproduced fingerprint and, in some cases, these alterations strongly impact on the final quality of the obtained image. Figure 2 shows some examples of fake fingerprint images where it can be easily observed the different visual quality. It is worth noting that no previous work has devoted much attention to this issue. However, in our opinion, it is important because adding a fake fingerprint detector obviously impacts on the false rejection rate of the system, that is, the rate of genuine rejected due to misclassified live fingerprints. Fake fingerprint images of poor quality, as those showed in Figure 2(a, c), could be easily rejected without employing a fake detector. It is worth noting that this problem arises independently on the position of the fake detection module into the processing chain (e.g. if the fake detection is done before or after the verification stage).

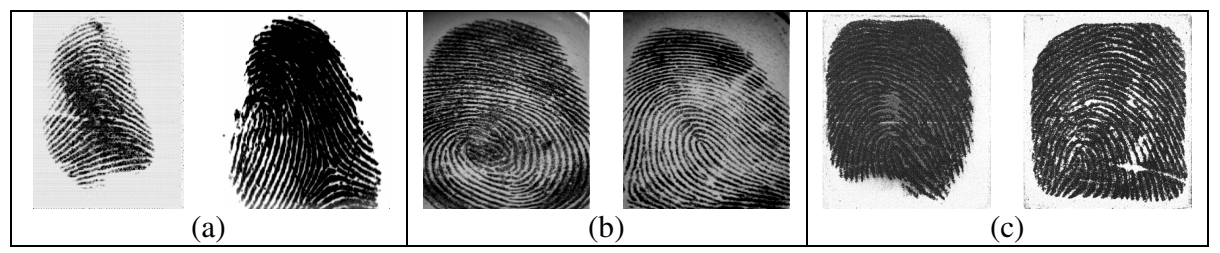

Fig. 2. Examples of fake fingerprint images from Ref. [9] (a), Ref. [14] (b), Ref. [11] (c)

The second item we raised in this Section concerns the characteristics of the data set used for the vitality detection experiments. Table 2 points out the most important characteristics of the data sets used in previous works. The second column reports the number of different fake fingerprints (subjects), the third the number of impressions for each fingerprint, the fourth the number of image frames acquired. The fifth column points out if the subjects used for producing stamps are the same used as clients, namely, if the data set contains both fake and live fingerprint images for each individual.

Information reported in Table 2 is useful to analyze: (1) the sample size of data sets for fake detection rate evaluation; (2) the protocol adopted in experiments.

With regard to item (1), it is worth noting that it requires several resources in terms of volunteers, time, and personnel devoted to stamp fabrication. In particular, volunteers must be trained to appropriately press their finger on the mould material, and a visual analysis of the stamp is necessary in order to obtain good quality images. In order to produce an acceptable stamp, many trials are required. Since the solidification of the mould material can require several hours, this impacts on the number of fake stamps produced per time unit. As a consequence, reported experimental results can be affected by the small sample size of the used data set. This could cause a not reliable estimation of the detection performance. 
Table 2. Some key characteristics of the data set used for vitality detection experiments in previous works

\begin{tabular}{|l|l|l|l|l|}
\hline Reference & No. fakes & No. impressions & No. frames & $\begin{array}{l}\text { Correspondence } \\
\text { with clients ? }\end{array}$ \\
\hline$[9]$ & 18 & 1 & 2 & NO \\
\hline$[10]$ & 32 & 10 & 0 & YES \\
\hline$[11]$ & 24 & 1 & 0 & NO \\
\hline$[13]$ & 12 & 5 & 20 & NO \\
\hline$[12]$ & 33 & 1 & 2 & NO \\
\hline$[14]$ & 28 & 2 & 2 & YES \\
\hline$[15]$ & 80 & 1 & 0 & NO \\
\hline
\end{tabular}

The differences about the above item (2), i.e., the differences of the characteristics of the data sets, are pointed out by the fifth column of Table 2. The term "correspondence with client" means that for each fake image, there is the correspondent live image. This impacts on the experimental protocol used and the final goal of the experimentation. In particular, works for which this correspondence is absent are aimed to point out that the proposed feature(s) can allow to distinguish a fake image from an alive one. Accordingly, they do not require the presence of related clients. On the other hand, they do not allow evaluating the penetration rate of the stamps in a verification system, that is, to assess the rate of fake fingerprints which would be accepted as live fingerprints.

In some cases (Table 2, third and sixth rows), a certain number of live and fake fingerprint frames/impressions is captured from the same subject. This is due to the characteristic of the measure, which requires the comparison of the input impression with the related template client (e.g. elastic distortion or morphological measures [10, 14] which require an additional minutiae extraction step), and also to the possibility of evaluating the relationship between fake detection rate and verification performance. In this case the protocol adopted is a little more complex, because the fake detection features can be extracted only by the comparison phase. As an example, the protocol adopted in [14] is made up of the following steps:

- the second impression has been considered as the template of the fingerprint stored in the system database. The minutiae-points were manually detected in order to avoid errors due to the minutiae detection algorithm;

- the first and the second frame of the first impression have been considered as the images provided by the system during an access attempt. Only attempts related to fingerprints of the same subject were considered ("genuine" attempts by live and fake fingers). Even for these images the minutiae-points were manually detected.

\section{Previous Works: Vitality Detection Performances}

Table 3 reports a preliminary comparison of previous works in terms of overall missdetection (classification) rate, that is, the average between the rate of "live" fingerprints wrongly classified as fake ones and viceversa.

Due to several problems, as the differences in terms of sensors used, data set size, protocol, and classifiers, it is difficult to fairly compare these values. As an example, 
the work by Antonelli et al. [13] uses a threshold-based classifier based on the monodimensional distribution of live and fake classes. This distribution has been computed by the Euclidean distance between template and input distortion codes. The threshold has been tuned on the "equal error rate" value. This value is obviously different from the overall error rate usually considered for evaluating the performance of vitality detection systems.

Moreover, the strong variations in error rates even when using similar approaches (e.g. see [10] and [14], or [9] and [12]) suggest that a common experimental protocol is necessary in order to avoid the difficulty in interpreting reported results.

Finally, an average error rate of 3-5\% even in small data sets makes these systems quite unacceptable for a real integration in current fingerprint verification systems, due to their impact on the false rejection rates (i.e. wrongly rejected clients) which could increase. As an example, the best fingerprint verification system at the 2004 edition of Fingerprint Verification Competition exhibited a $2 \%$ equal error rate on average. [16]

Table 3. Vitality detection performance reported in previous works

\begin{tabular}{|l|l|l|}
\hline Reference & Classification method & Error rate \\
\hline$[9]$ & Back-propagation neural network & $0 \%$ \\
\hline$[10]$ & Support Vector Machine & $18 \%$ \\
\hline$[11]$ & Threshold & $0 \%$ \\
\hline$[13]$ & Threshold & $5 \%$ (equal error rate) \\
\hline$[12]$ & Neural Network, Threshold, & $6 \%$ (capacitive sensor) \\
& Linear Discriminant Analysis & $3 \%$ (electro-optical sensor) \\
& & $2 \%$ (optical sensor) \\
\hline$[14]$ & k-NN Classifier & $6 \%$ \\
\hline$[15]$ & Classification Tree & $5 \%$ (capacitive sensor) \\
& & $13 \%$ (optical sensor) \\
\hline
\end{tabular}

\section{Conclusions}

Fingerprint vitality detection has become a crucial issue in personal verification systems using this biometric.

In this paper, we critically reviewed the main approaches to fingerprint vitality detection proposed in these years. To the best of our knowledge, this is the first survey about fake fingerprint detection methods. In particular, we proposed a possible taxonomy for summarizing the current state-of-the-art and also examined the scientific literature from other point of views, as the materials employed for producing fake stamps and the data sets used for experiments. Finally, we reported the detection rates of previous works in order to trace a preliminary comparison among the current approaches.

Our future work will analyze further the state of the art and will also perform a fair experimental comparison by adopting an appropriate data set aimed to highlight pros and cons of the state-of-the-art methods for fingerprint vitality detection. 


\section{References}

[1] Maltoni, D., Maio, D., Jain, A.K., Prabhakar, S. (eds.): Handbook of fingerprint recognition. Springer, Heidelberg (2003)

[2] Bolle, R.M., Connell, J.H., Ratha, N.K.: Biometric perils and patches. Pattern Recognition 35(12), 2727-2738 (2002)

[3] Matsumoto, T., Matsumoto, H., Yamada, K., Hoshino, H.: Impact of artificial 'gummy' fingers on fingerprint systems. In: Proceedings of SPIE, vol. 4677 (2002)

[4] Lapsley, P., Less, J., Pare, D., Hoffman, N.: Anti-Fraud Biometric Sensor that Accurately Detects Blood Flow, SmartTouch, LLC, US Patent \#5,737,439 (1998)

[5] Biel, L., Pettersson, O., Philipson, L., Wide, P.: ECG analysis: A new approach in human identification. IEEE Transactions on Instrumentation and Measurement 50(3), 808-812 (2001)

[6] Baldissera, D., Franco, A., Maio, D., Maltoni, D.: Fake Fingerprint Detection by Odor Analysis. In: proceedings International Conference on Biometric Authentication (ICBA06), Hong Kong (January 2006)

[7] Osten, D., Carim, H.M., Arneson, M.R., Blan, B.L.: Biometric, Personal Authentication System, U.S. Patent \#5 719950 (February 17, 1998)

[8] Jain, A.K., Bolle, R., Pankanti, S. (eds.): BIOMETRICS: Personal Identification in Networked society. Kluwer Academic Publishers, Dordrecht (1999)

[9] Derakhshani, R., Schuckers, S., Hornak, L., O’Gorman, L.: Determination of vitality from a non-invasive biomedical measurement for use in fingerprint scanners. Pattern Recognition 36(2), 383-396 (2003)

[10] Chen, Y., Jain, A.K., Dass, S.: Fingerprint deformation for spoof detection. In: Biometric Symposium, Cristal City, VA (2005)

[11] Moon, Y.S., Chen, J.S., Chan, K.C., So, K., Woo1, K.C.: Wavelet based fingerprint liveness detection. Electronics Letters 41(20), 1112-1113 (2005)

[12] Parthasaradhi, S., Derakhshani, R., Hornak, L., Schuckers, S.: Time-series detection of perspiration as a vitality test in fingerprint devices. IEEE Trans. On Systems, Man and Cybernetics, Part C 35(3), 335-343 (2005)

[13] Antonelli, A., Cappelli, R., Maio, D., Maltoni, D.: Fake Finger Detection by Skin Distortion Analysis. IEEE Transactions on Information Forensics and Security 1(3), 360 373 (2006)

[14] Coli, P., Marcialis, G.L., Roli, F.: Analysis and selection of feature for the fingerprint vitality detection. In: SSPR/SPR 2006, pp. 907-915 (2006)

[15] Tan, B., Schuckers, S.: Liveness detection for fingerprint scanners based on the statistics of wavelet signal processing. In: Conference on Computer Vision Pattern Recognition Workshop (CVPRW'06) (2006)

[16] Maio, D., Maltoni, D., Cappelli, R., Wayman, J.L., Jain, A.K.: FVC2004: Third Fingerprint Verification Competition. In: Zhang, D., Jain, A.K. (eds.) ICBA 2004. LNCS, vol. 3072, pp. 1-7. Springer, Heidelberg (2004) 